\title{
Ocular Manifestations of Donnai Barrow Syndrome
}

\author{
Ayoub Redouan, Hind Hamdani,Amrani Soukaina, Loubna El Maaloum, \\ Bouchra Allali, and Asmae El Kettani
}

ABSTRACT

Donnai-Barrow syndrome is an autosomal recessive disease due to mutations in the LRP2 gene described by Donnai and Barrow in 1993. We report through this clinical case the various ophthalmologic and extraocular manifestations of Donnai Barrow syndrome which remains a very rare congenital malformation. High myopia is a risk factor for severe amblyopia and/or retinal detachment which requires a regular monitoring.

The management of this disabling disease is multidisciplinary, involving the ophthalmologist, the otorhinolaryngologist, the pediatrician and the psychiatrist.

Keywords: Donnai-Barraow syndrome, high myopia, amblyopia, retinal detachment.

Published Online: December 16, 2020

ISSN: $2593-8339$

DOI: 10.24018 /ejmed.2020.2.6.605

Ayoub Redouan*

Hassan II University, Morocco.

(e-mail: drayoubredouan ${ }^{\circledR}$ gmail.com)

Hind Hamdani

Hassan II University, Morocco.

Soukaina Amrani

Hassan II University, Morocco.

Loubna EI Maaloum

Hassan II University, Morocco.

Bouchra Allali

Hassan II University, Morocco.

Asmae El Kettani

Hassan II University, Morocco.

*Corresponding Author

\section{INTRODUCTION}

Donnai-Barrow Syndrome (DBS) is a rare multiple congenital malformation first described by Donnai and Barrow, combining a characteristic facial dysmorphia, ocular disorders, deafness, congenital diaphragmatic hernia, and variable intellectual deficit. We report the case of a 2-yearold child, referred for an ophthalmological examination under general anesthesia, for a poor visual behaviour noticed by the mother. The diagnosis of DBS was retained in front of the typical facial dysmorphia, congenital diaphragmatic hernia and strong myopia. In the light of this observation, we describe the ocular and extra-ocular manifestations of this syndrome, as well as the importance and the benefits of a genetic and prenatal diagnosis.

\section{CASE REPORT}

We report the case of a 2-year-old only child, from a firstdegree consanguineous marriage, born at term, with a birth weight of $2.75 \mathrm{~kg}$. Clinical examination found an umbilical hernia (Fig. 1) associated with facial dysmorphia including a large and broad forehead, hypertelorism, oblique palpebral slits down and out and a short nose with a flat nasal bridge (Fig. 2). Ophthalmologic examination under anesthesia found high myopia at -10 diopters in the right eye and -11 diopters in the left eye, a clear cornea with a corneal diameter of 11 $\mathrm{mm}$ in both eyes, the intraocular pressure measured by Perkins tonometer was $8 \mathrm{mmhg}$ right eye and $10 \mathrm{mmhg}$ left eye. Examination of the posterior segment revealed a diffuse chorio-retinal atrophy in both eyes with a normal macula and papilla without retinal detachment. A karyotype study with
400 base pair resolution was performed and was proved to be normal.

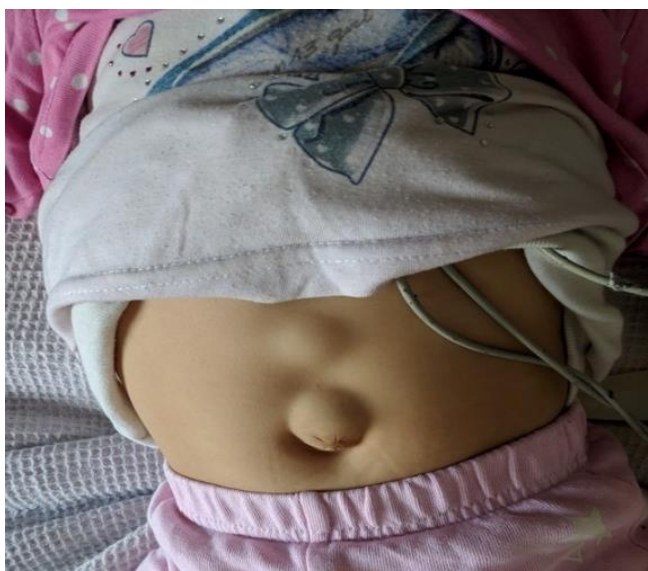

Fig. 1. Aspect of umbilical hernia

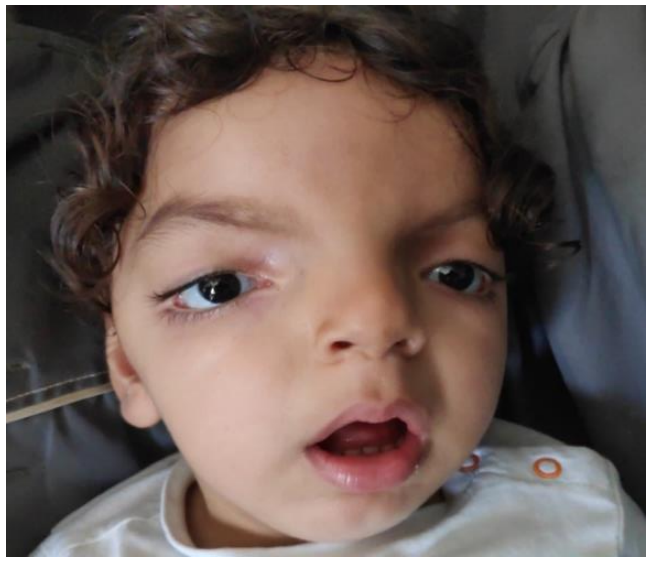

Fig. 2. Facial dysmorphia characteristics: hypertelorism, down slanting, palpebral fissures and short nose. 
The MRI revealed a hypogenesis of the corpus callosum (Fig. 3). The patient had an auditory evoked potential which revealed a profound sensorineural hearing loss for which a cochlear implant was indicated (Fig. 4). The patient received an optical correction and benefited from a complete ophthalmological examination under sedation every 6 months (retinal periphery, measurement of intraocular pressure, anterior segment, and refraction under cycloplegia).

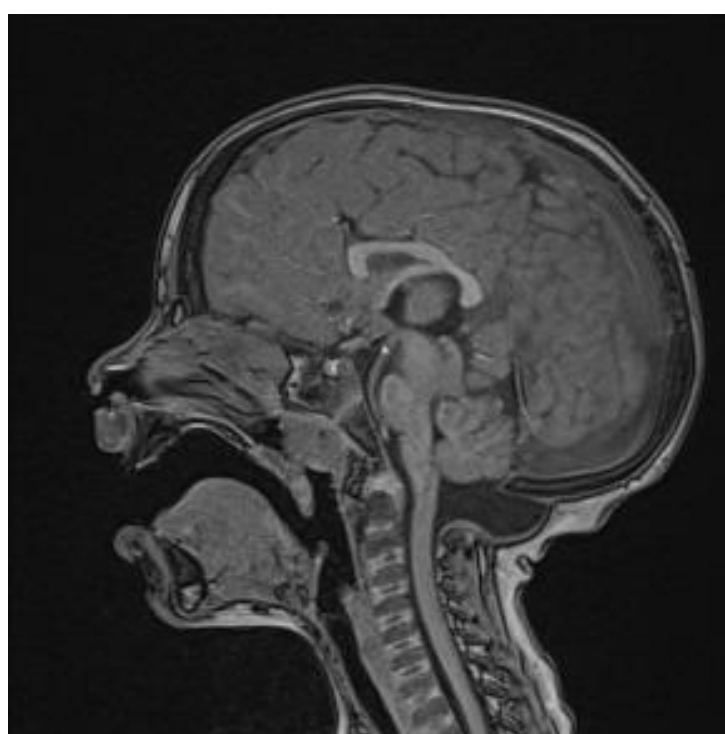

Fig. 3. A sagittal view of MRI of the brain, shows a hypogenesis of the Corpus callosum.

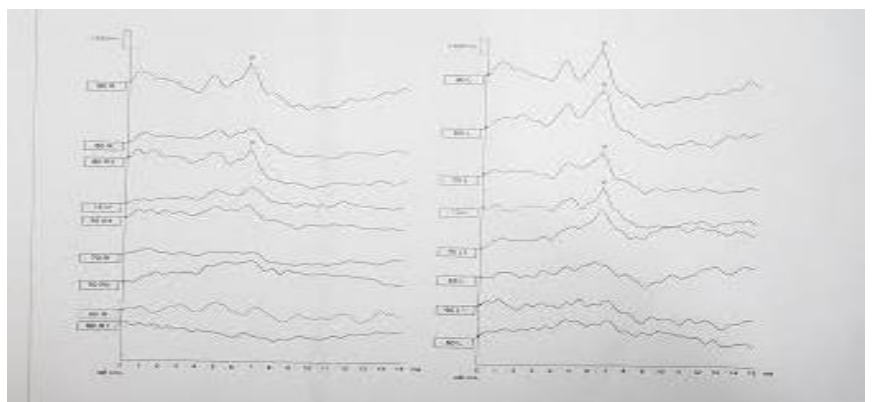

Fig. 4. An auditory evoked potential reveals a profound bilateral sensorineural hearing loss.

\section{DISCUSSION}

Donnai-Barrow syndrome is a rare birth defect first described in 1993 by Donnai and Barrow [1]. It is an autosomal recessive disease [2] due to mutations in the LRP2 gene (2q31.1) which codes for megalin [3]. This protein is highly expressed on the surface of the cerebral absorption epithelium, the optic cup, the optic placode and the proximal convoluted tubule. It is involved in the recapture of many proteins in the proximal convoluted tubule, including retinol binding proteins (RBP4) and vitamin D binding proteins (VDBP) [4]. The absence of megalin results in tubular proteinuria with excessive leakage of low molecular weight proteins (RBP4, VDBP), contributing to the typical clinical manifestations of Donnai-Barrow syndrome [5].

Corpus callosum hypogenesis, profound sensorineural deafness, facial dysmorphia and severe myopia are present in all patients, as reported in our case, while diaphragmatic hernia and omphalocele in $40 \%$ of patients [6,] [7].
Facial dysmorphia is characteristic: an enlargement of the anterior fontanel, a large and broad forehead, hypertelorism, a short nose with a flat nasal bridge and oblique palpebral slits down and out [8]. On the ophthalmological side, a refraction under cycloplegia allow us to diagnose a high axial myopia, which must be corrected as early as possible with regular monitoring to avoid amblyopia. Coloboma, which is often bilateral, and/or iris hypoplasia, congenital cataract and glaucoma are not commonly associated with this syndrome [2], [6]. Retinal detachment is quite frequent (30\%). It can be either unilateral or bilateral, with or without macular detachment. Hence the interest of a prophylactic barrage laser of the retinal periphery [6], [7]. Besides, rarely cases reported of Donnai-Barrow syndrome associated with a hypoplasic optic nerve, a small optic head nerve and optic head nerve pigmentary abnormalities.

The parents of a child with Donnai-Barrow syndrome are necessarily carriers of the allele that expresses this disease, they are called heterozygous. Therefore, genetic counseling is very important. It consists in explaining to the parents the autosomal recessive mode of transmission of the disease, and the probabilities of expressing the different clinical manifestations of the disease during future pregnancies [2]. Thus, DBS gene carrier can benefit from a prenatal testing of their pregnancies, as well as their relatives.

\section{CONCLUSION}

We report through this clinical case the various clinical manifestations of Donnai Barrow syndrome which remains a rare pathology. Strong myopia is a risk factor for low vision that requires careful and regular monitoring. Treatment by laser photocoagulation of the retinal periphery should not be systematic despite the risk of retinal detachment. The patients must benefit from multidisciplinary care (ophthalmologist, otorhinolaryngologist, pediatrician and psychiatrist) for a better quality of life.

\section{REFERENCES}

[1] Donnai D, Barrow M. Diaphragmatic hernia, exomphalos, absent corpus callosum, hypertelorism, myopia, and sensorineural deafness: a newly recognized autosomal recessive disorder. Am J Med Genet 1993;47:679e82.

[2] Kantarci S, Donnai D, Noonan KM et al. Donnai Barrow Syndrome GeneReviews at Genetets. 1993-2008. Available at http:// www.genetests.org. Accessed August 28, 2010.

[3] Stora S, Conte M, Chouery E, Richa S, Jalkh N, Gillart A-C, Joannis AL, Mégarbané A. A 56-year-old female patient with facio-oculoacoustico-renal syndrome (FOAR) syndrome. Report on the natural history and of a novel mutation. Eur J Med Genet. 2009 Oct;52(5):34143.

[4] Assemat E, Chatelet F, Chandellier J, et al. Overlapping expression patterns of the multiligand endocytic receptors cubilin and megalin in the CNS, sensory organs, and developing epithelia of the rodent embryo. Gene Expr Patterns 2005; 6:69-78

[5] Anglani F, Terrin L, Brugnara $M$ et al. Hypercalciuria and nephrolithiasis: expanding the renal phenotype of Donnai-Barrow syndrome. Clin Genet. 2018; 94:187-8.

[6] Patel N, Hejkal T, Katz A, Margalit E. Ocular manifestations of Donnai-Barrow syndrome. J Child Neurol. $2007 ; 22: 462-4$.

[7] Mauro Longoni, Sibel Kantarci, Dian Donnai et al. Donnai-Barrow Syndrome. Gene Reviews. 20301732.

[8] Pober BR, Longoni M, Noonan KM. A review of Donnai-Barrow and facio-oculo-acoustico-renal (DB/FOAR) syndrome: clinical features and differential diagnosis. Birth Defects Res A Clin Mol Teratol. 2009; 85:76-81 